\title{
Inflammaging 2018: An update and a model
}

Claudio Franceschi ${ }^{\mathrm{a}, \mathrm{b}, \mathrm{c}, *}$

${ }^{a}$ Department of Experimental, Diagnostic and Specialty Medicine, University

of Bologna, Italy

${ }^{\mathrm{b}}$ IRCCS Institute for Neurological Sciences, Bellaria Hospital, Bologna, Italy

${ }^{\mathrm{c}}$ Department of Applied Mathematics and Laboratory of Systems Biology of Aging, Lobachevsky University, Nizhny Novgorod, Russia

E-mail address: claudio.franceschi@unibo.it.

Alexey Zaikin ${ }^{\text {a,b }}$

${ }^{\mathrm{a}}$ Department of Applied Mathematics and Laboratory of Systems Biology of Aging, Lobachevsky University, Nizhny Novgorod, Russia

${ }^{\mathrm{b}}$ University College London, United Kingdom

Susanna Gordleeva, Mikhail Ivanchenko

Department of Applied Mathematics and Laboratory of Systems Biology of Aging, Lobachevsky University, Nizhny Novgorod, Russia

Francesca Bonifazi

Hematology Department, “L \& A Seragnoli Institute, S.Orsola-Malpighi Hospital, Bologna, Italy

of Bologna, Italy Istituto Scientifico Romagnolo per lo Studio e la Cura dei Tumori (IRST) IRCCS, Meldola, Italy

Gianluca Storci $^{1}$

Department of Experimental, Diagnostic and Specialty Medicine, University of Bologna, Italy

Massimiliano Bonafè a,b,1

${ }^{\text {a }}$ Department of Experimental, Diagnostic and Specialty Medicine, University

${ }^{\mathrm{b}}$ Istituto Scientifico Romagnolo per lo Studio e la Cura dei Tumori (IRST) IRCCS, Meldola, Italy 


\section{Inflammaging: the state of the art}

'Inflammaging' refers to the chronic, low-grade inflammation that characterizes aging. The conceptualization of inflammaging, resulting from the convergence of studies on the evolution of immune system from invertebrates to vertebrates and studies on immunosenescence in humans and particularly in centenarians $[1,2]$ was first heralded in 2000 to underpin the intimate relationship between aging and the life- long activation of the inflammatory response [3]. This tenet forewent the more recent approach of Geroscience that identifies inflammation as one of the seven pillars of aging (adaptation to stress, epigenetics, macromolecular damage, metabolism, proteostasis, stem cells and re- generation and inflammation) [4]. The new perspective of Geroscience suggests that aging is the most important risk factor for age-related diseases (ARDs) and geriatric syndromes (GSs), and that aging and ARDs/GSs share the same basic molecular mechanisms (the abovementioned pillars), including chronic inflammation/inflammaging [5]. Moreover, a large literature suggests that alterations of the pillars converge on chronic inflammation/inflammaging which likely re- present the most common output.

Accordingly, we argued that ARDs and GSs can be conceptualized as manifestations of accelerated aging. In particular, clinically different ARDs/GSs are the result of peculiar combinations of alterations that regard the same limited set of basic mechanisms, including inflamma- ging [6]. Whether an individual will follow a trajectory of accelerated or decelerated aging will depend on his/her genetic background in- teracting lifelong with environmental and lifestyle factors (nutrition, physical and mental activity, among others) [7]. As ARDs and GSs are manifestations of accelerated aging, it is urgent to identify markers capable of distinguishing between biological and chronological age in order to identify subjects at higher risk of developing ARDs and GSs. To this aim, we have proposed the use of DNA methylation [8], N-glycans profiling [9] and gut microbiota composition [10] to complement the available disease-specific markers [7]. Aging/inflammaging appear to be the evolutionary-unpredicted consequence of the "degeneracy" of the damage sensors, whose capability to distinguish between self and non-self and to mount an inflammatory response is blurred in verte- brates and even more in old subjects [7]. Overall, aging/inflammaging can be conceptualized as autoimmune/autoinflammatory phenomena/ processes [2], characterized, among others, by the presence of auto- antibodies that likely help in getting rid of molecular and cellular by- products [11]. Intriguingly, centenarians are characterized by high levels non-organ specific autoantibodies (e.g. anti-poly ADP-ribose polymerase) supporting the concept of "protective autoantibodies" that may bind to senescent (self) neo-antigens and facilitate their catabo- lism, as originally suggested by Pierre Grabar (1898-1986) [11].

According to this integrated view, aging and ARDs/GSs become part of a continuum [6] where precise boundaries do not exist, and the two extremes are represented by centenarians [12-14] and their offspring [15] who largely avoided or postponed most ARDs/GSs and are char- acterized by decelerated aging [16], and patients who suffered one or more severe ARDs/GSs in their $60 \mathrm{~s}, 70 \mathrm{~s}$, and $80 \mathrm{~s}$ and show signs of accelerated aging, respectively [7]. In between these two extremes, there is a continuum of intermediate trajectories representing a sort of gray area. In the past twenty years, the concept of inflammaging has shed light on such grey zone of systemic inflammation in aged people, a condition that spans from the healthy aging status to overt inflammatory ARDs/GSs. In turn, inflammaging is accelerated by persis- tent infections (cytomegalovirus) [17], lifestyle habits such as nutrient excess (metaflammation and obesity) [7,18], physical and psychosocial/emotional stressors $[19,20]$ and gut microbiota dysbiosis [10].

The last conceptual development of this inflammatory theory of aging suggests that the most important/causal inflammatory stimuli fueling inflammaging are to be identified in the age-related dysregu- lation of the production of endogenous "molecular garbage" [2]. The central tenets are the following: i) molecular garbage is continuously and physiologically produced as a consequence of cell death (ne- croptosis, pyroptosis, netosis), metabolism [7] and gut microbiota function [10], but also continuously neutralized by the remodeling and adaptive capability of the body (e.g. production of anti-inflammatory molecules) which quickly and efficiently 
down-regulate inflammatory responses in young subjects [21]; ii) molecular garbage continuously stimulates an innate immunity-driven inflammatory response by mac- rophages with the participation of other cells belonging to adaptive immunity; iii) the balance between garbage generation and disposal becomes impaired with age; iv) age-related mitochondrial dysfunction contributes to inflammaging as a source of oxidative stress [22] and 'selfgarbage' (mtDNA, cardiolipin, or formyl peptides) that can be sensed by macrophages ; $\mathbf{v}$ ) a variety of potentially inflammatory self- molecules (including nuclear DNA, misplaced molecules, cell debris produced as a consequence of cell death and other cellular garbage and signaling molecules such as miRNAs) freely circulate in body fluids within extracellular vesicles/exosomes carrying inflammatory signals that can spread to distal cells and tissues, thus propagating inflamma- ging and aging; vi) human aging/inflammaging as well as human longevity and their genetics are highly context-dependent, dynamic processes/phenomena both historically and individually $[14,23,24]$.

\section{Inflammaging: the new advances}

The contributions presented in this issue of Seminars in Immunology address critical topics which altogether widen the above- mentioned scenario and propose an integrated new view of inflamma- ging, less simplistic than previously thought, and stress the capability of this theory to identify new targets of intervention, thus contributing the field of translational medicine of the elderly.

As far as the Geroscience-predicted deep intertwining between aging/inflammaging and ARDs/GSs, Fulop et al., argue that studies on inflammaging are expected to provide clues on the mechanisms that underlay both the aging process and the pathogenesis of age-related diseases. Moreover, as outlined by the same authors, the clinical eva- luation of inflammaging and the assessment of the cognate biochemical parameters are expected to improve our understanding on the health status of the elderly patient. So far, the molecular basis of inflammaging is still to be clarified and may ensue different overlapping mechanisms.

As far as the conceptualization of inflammaging and garbaging, Storci et al., suggest that nucleic acids released outside their native cellular compartments are major fuel of inflammaging and the related chronic activation of the innate immune system. The authors underpin the concept that changes with age in the biochemical milieu of such nucleic acid burden content (e.g. cytosine methylation, RNA:DNA hy- brids, G-rich DNA sequences, mtDNA) affect its capability to elicit chronic inflammation. In this potentially pro-inflammatory scenario, enzymes such as DNAses and RNAseH continuously degrade nucleic acid fragments while the telomere exerts a potent anti-inflammatory activity.

The contribution of Bottazzi et al., follows the same direction, and it is focused on the relationship between cancer and inflammaging. The authors argue that also in cancer research for many decades the at- tention of epidemiologists was attracted by environmental exogenous carcinogens and that only relatively recently the cancer inflammatory microenvironment, dominated by endogenously-produced in- flammatory stimuli and the struggle between a number of pro- and anti- inflammatory cell types, was considered a critical issue to understand progression and metastasis [25].

Indeed, according to the importance for health and disease of an optimal the balance between inflammaging and anti-inflammaging, as proposed in 2007 [21], Doyle et al., focus on resolvins (lipoxins, re- solvins, protectins and maresins) and argue that a modern view of in- flammaging must be comprehensive of the process of resolution, i.e. the wealth of mechanisms that actively switch off the inflammatory re- sponse. Lacking/insufficient resolution machinery promotes the onset of chronic inflammation and is likely to unbalance the above-men- tioned delicate equilibrium between inflammaging and anti-in- flammaging [21].

Two basic mechanisms of aging highlighted by Geroscience [4], i.e. epigenetics and inflammasome activation are addressed by Nardini et al., and Duewell et al., respectively. 
Nardini et al., describe current knowledge on the reciprocal re- lationship between inflammaging and the remodelling of epigenetic modifications. This is an emerging topic of interest, not only because it can contribute to the characterization of the molecular basis of in- flammaging, but also because it can provide new aging biomarkers. Furthermore, the authors argue that as epigenetic patterns can be modulated by the exposure to environmental and nutritional stimuli, the study of the epigenetics of inflammaging can provide new modifiable targets to counteract chronic inflammation in the elderly.

According to Duewell et al., inflammasomes have been identified as important drivers of sterile inflammation and that there is accumulating evidence that NLRP3 activation, because of its broad variety of acti- vating agents, may play an important role during the aging process (inflammaging). Since endogenous triggers activate the inflammosome pathway is likely to be critical for the development of metabolic dis- eases. Accordingly, NLRP3 inhibition reduces systemic inflammation, reduced immune cell activation and improves insulin sensitivity. Thus, it is tempting to speculate that inflammasomes serve as key upstream integrators of the innate immunesystems to drive the inevitable process of inflammaging. Finally, the authors argue that inflammasome activation in the hematopoietic system is involved in the accumulation with age, up to the limits of human lifespan, of HSC clones that carry specific genetic alterations and generate TET2-deficient macrophages that have an increased expression of NLRP3 and promote the growth of the atherosclerotic plaque.

As far as the translational side of inflammaging is concerned two papers review the application of inflammaging to the problems related to the failure to vaccinate a consistent number of old subjects towards a variety of vaccines (Ciabattini et al.,) and to the role of immune system in getting rid of senescent cells, a major producers of inflammatory compounds (Langhi et al.,).

Ciabattini et al., suggest that the use of concepts such as "in- flammaging", "immunobiography" and "biological age" could and should be applied to vaccinology to go beyond the present conceptual framework mostly adopted in this field so important for the health of the elderly at the population level. As reviewed by Langhi et al., cell senescence is a cellular program that generates pro-inflammatory cells in response to a variety of damaging stimuli. Recent literature shows that the ablation of senescent cells, that constitute a minor population in aged tissues, is sufficient to restore functional/anatomical re- juvenation, thus demonstrating that few (senescent) cells can take-over the homeostatic control of an entire tissue. These data therefore suggest that the more knowledge on the senescence program will be generated, the better strategies to combat aging will be unraveled.

\section{Inflammaging: a new model}

The above-mentioned evidence lead to hypothesize a contribution of senescent cells to inflammaging and its propagation to neighboring cells and distant organs [2]. Accordingly, we here present a mathe- matical model of inflammaging based on available knowledge and the data and concepts developed in this issue. The model is focused on the bone marrow as an example of a tissue critical for the generation of immune cells including macrophages, and particular attention is de- voted to the age related expansion of clones carrying specific mutations (i.e. DNMT3A and TET2) and their capability to generate a pro-in- flammatory microenvironment. The phenomenon is paralleled by the accumulation of DNA mutations in stromal cells which activate proinflammatory DNA response and ultimately undergo senescence (Fig. 1). On the whole, the main message of the proposed inflammaging model is that in a tissue such as the bone marrow the aging process leads to an overpowering of pro-inflammatory cells as a consequence of the converge of the different cell types to a proinflammatory pheno- type (decreased cell heterogeneity + clonal expansions), which favors the propagation of the inflammatory wave. In this regard, the mathe- matical model proposed in Fig. 2 shows that phenotypic heterogeneity in a tissue is a barrier of inflammaging propagation while tissue homogeneity favors the propagation of the inflammatory wave. In the setting of the bone marrow this situation may be represented, by pre- dominance of proinflammatory HSC clones, stromal and myeloid cells (Fig. 2). The oligoclonal-drift of the $\mathrm{BM}$ is reminiscent of the fulfilling of the "immunological ecosystem" by expanded T oligo-clones, which at least 
in part, is due to the chronic attrition of antigen exposure (e.g. CMV infections) [26]. Similar phenomenon occurs to B cells in which the accumulation of dominant memory B clones in aged population is paralleled by the occurrence of monoclonal peaks in serum and by the expansion of clones that may react to autoantigens [27] (Fig. 3).

\section{Inflammaging: conclusions and perspectives}

We surmise that the future of inflammaging can be summarized as depicted in Fig. 4. Inflammaging as a pillar of the aging process and owing to its major role in the pathogenesis of ARDs and GSs has to be further investigated and developed according to two apparently oppo- site but in reality complementing directions represented by: i) the ex- ploitation of large datasets regarding a large number of subjects of different ages and health status, from fetuses and neonates to super- centenarians, and the use of artificial intelligence; ii) the in depth analysis of individuals, i.e. single persons, in order to catch the perso- nalized characteristics of inflammaging.

\section{Declaration of interest}

The authors declare to have no conflict of interest.

\section{Acknowledgments}

By Fondazione Cassa di Risparmio delle province Lombarde (CARIPLO, Rif. 2015-0546 and 2016-0835 to CF) by the Seventh fra- mework programme (Grant number 602757, HUMAN), by the Horizon 2020 framework programme (Grant number 634821, PROPAG- AGING), by JPco-Fund (ADAGE) to CF; Pallotti Foundation to MB; Ministry of Education and science of the Russian federation agreement n ${ }^{\circ}$ 074-02-2018-330 to CF, MI, AZ and SG.

\section{References}

[1] C. Franceschi, D. Monti, P. Sansoni, A. Cossarizza, The immunology of exceptional individuals: the lesson of centenarians, Immunol. Today 16 (1995) 12-26.

[2] C. Franceschi, P. Garagnani, G. Vitale, M. Capri, S. Salvioli, Inflammaging and' Garb-aging', Trends Endocrinol. Metab. 28 (2017) 199212 .

[3] C. Franceschi, M. Bonafè, S. Valensin, F. Olivieri, M. De Luca, E. Ottaviani, G. De Benedictis, Inflamm-aging. An evolutionary perspective on immunosenescence, Ann. N. Y. Acad. Sci. 908 (2000) 244-254.

[4] B.K. Kennedy, S.L. Berger, A. Brunet, J. Campisi, A.M. Cuervo, E.S. Epel, C. Franceschi, G.J. Lithgow, R.I. Morimoto, J.E. Pessin, T.A. Rando, A. Richardson, E.E. Schadt, T. Wyss-Coray, F. Sierra, Geroscience: linking aging to chronic disease, Cell 159 (2014) $709-713$.

[5] C. Franceschi, J. Campisi, Chronic inflammation (inflammaging) and its potential contribution to age-associated diseases, J. Gerontol. A Biol. Sci. Med. Sci. 69 (Suppl) (2014) S4-9.

[6] C. Franceschi, P. Garagnani, C. Morsiani, M. Conte, A. Santoro, A. Grignolio, D. Monti, M. Capri, S. Salvioli, The continuum of aging and age-related diseases: common mechanisms but different rates, Front. Med. (Lausanne) 5 (2018) 61.

[7] C.Franceschi,P.Garagnani,P.Parini,C.Giuliani,A.Santoro,Inflammaging:anew immune-metabolic viewpoint for age-related diseases, Nat. Rev. Endocrinol. 14 (2018) 576-590.

[8] P. Garagnani, M.G. Bacalini, C. Pirazzini, D. Gori, C. Giuliani, D. Mari, A.M. Di Blasio, D. Gentilini, G. Vitale, S. Collino, S. Rezzi, G. Castellani, M. Capri, S. Salvioli, C. Franceschi, Methylation of ELOVL2 gene as a new epigenetic marker of age, Aging Cell 11 (2012) $1132-$ 
1134.

[9] F. Dall'Olio, V. Vanhooren, C.C. Chen, P.E. Slagboom, M. Wuhrer, C. Franceschi, N- glycomic biomarkers of biological aging and longevity: a link with inflammaging, Ageing Res. Rev. 12 (2013) 685-698.

[10] E. Biagi, C. Franceschi, S. Rampelli, M. Severgnini, R. Ostan, S. Turroni, C. Consolandi, S. Quercia, M. Scurti, D. Monti, M. Capri, P. Brigidi, M. Candela, Gut microbiota and extreme longevity, Curr. Biol. 26 (2016) 1480-1485.

[11] M.E. Weksler, G. Pawelec, C. Franceschi, Immune therapy for age-related diseases, Trends Immunol. 30 (2009) 344-350.

[12] C. Giuliani, C. Pirazzini, M. Delledonne, L. Xumerle, P. Descombes, J. Marquis, G. Mengozzi, D. Monti, D. Bellizzi, G. Passarino, D. Luiselli, C. Franceschi, P. Garagnani, Centenarians as extreme phenotypes: an ecological perspective to get insight into the relationship between the genetics of longevity and age-associated diseases, Mech. Ageing Dev. 165 (2017) 195-201.

[13] C. Franceschi, R. Ostan, A. Santoro, Nutrition and inflammation: are centenarians similar to individuals on calorie-restricted like individuals? Annu. Rev. Nutr. 21 (2018) 329-356.

[14] C. Giuliani, P. Garagnani, C. Franceschi, Genetics of Human Longevity Within an Eco-Evolutionary Nature-Nurture Framework, Circ. Res. 123 (123) (2018) 745-772.

[15] P. Gueresi, R. Miglio, D. Monti, D. Mari, P. Sansoni, C. Caruso, E. Bonafede, L. Bucci, E. Cevenini, R. Ostan, M.G. Palmas, E. Pini, M. Scurti, C. Franceschi, Does the longevity of one or both parents influence the health status of their offspring? Exp. Gerontol. 48 (2013) 395-400.

[16] S. Horvath, C. Pirazzini, M.G. Bacalini, D. Gentilini, A.M. Di Blasio, M. Delledonne, D. Mari, B. Arosio, D. Monti, G. Passarino, F. De Rango, P. D’Aquila, C. Giuliani, E. Marasco, S. Collino, P. Descombes, P. Garagnani, C. Franceschi, Decreased epi- genetic age of PBMCs from Italian semi-supercentenarians and their offspring, Aging (Albany NY) 7 (2015) 1159-1170.

[17] P. Sansoni, R. Vescovini, F.F. Fagnoni, A. Akbar, R. Arens, Y.L. Chiu, L. Cičin-Šain, J. Dechanet-Merville, E. Derhovanessian, S. Ferrando-Martinez, C. Franceschi, D. Frasca, T. Fulöp, D. Furman, E. Gkrania-Klotsas, F. Goodrum, B. Grubeck- Loebenstein, M. Hurme, F. Kern, D. Lilleri, M. López-Botet, A.B. Maier, T. Marandu, A. Marchant, C. Matheï, P. Moss, A. Muntasell, E.B. Remmerswaal, N.E. Riddell, K. Rothe, D. Sauce, E.C. Shin, A.M. Simanek, M.J. Smithey, C. Söderberg-Nauclér, R. Solana, P.G. Thomas, R. van Lier, G. Pawelec, J. Nikolich-

Zugich, New advances in CMV and immunosenescence, Exp. Gerontol. 55 (2014) 54-62.

[18] C. Franceschi, Healthy ageing in 2016: Obesity in geroscience - is cellular senes- cence the culprit? Nat. Rev. Endocrinol. 13 (2017) 76-78.

[19] C.E. Hostinar, R. Nusslock, G.E. Miller, Future Directions in the Study of Early-Life Stress and Physical and Emotional Health: Implications of the Neuroimmune Network Hypothesis, J. Clin. Child Adolesc. Psychol. 47 (2018) 142-156.

[20] E. Ottaviani, C. Franceschi, The neuroimmunology of stress from invertebrates to man, Prog. Neurobiol. 48 (1996) 421-440.

[21] C. Franceschi, M. Capri, D. Monti, S. Giunta, F. Olivieri, F. Sevini, M.P. Panourgia, L. Invidia, L. Celani, M. Scurti, E. Cevenini, G.C. Castellani, S. Salvioli, Inflammaging and anti-inflammaging: a systemic perspective on aging and long- evity emerged from studies in humans, Mech. Ageing Dev. 128 (2007) 92-105.

[22] G. Vitale, S. Salvioli, C. Franceschi, Oxidative stress and the ageing endocrine system, Nat. Rev. Endocrinol. 9 (2013) $228-240$.

[23] A.I. Yashin, G. De Benedictis, J.W. Vaupel, Q. Tan, K.F. Andreev, I.A. Iachine, M. Bonafe, M. DeLuca, S. Valensin, L. Carotenuto, C. Franceschi, Genes, demo- graphy, and life span: the contribution of demographic data in genetic studies on aging and longevity, Am. J. Hum. Genet. 65 (1999) 1178-1193.

[24] C. Franceschi, P. Garagnani, Suggestions from geroscience for the genetics of age- related diseases, PLoS Genet. 12 (2016) e1006399. 
[25] M. Bonafè, G. Storci, C. Franceschi, Inflamm-aging of the stem cell niche: breast cancer as a paradigmatic example: breakdown of the multishell cytokine network fuels cancer in aged people, Bioessays 34 (2012) 40-49.

[26] C. Franceschi, M. Bonafè, Centenarians as a model for healthy aging, Biochem. Soc. Trans. 31 (2003) 457-461.

[27] C. Wang, Y. Liu, L.T. Xu, K.J. Jackson, K.M. Roskin, T.D. Pham, J. Laserson, E.L. Marshall, K. Seo, J.Y. Lee, D. Furman, D. Koller, C.L. Dekker, M.M. Davis, A.Z. Fire, S.D. Boyd, Effects of aging, cytomegalovirus infection, and EBV infection on human B cell repertoires, J.

Immunol. 192 (2014) 603-611.

[28] C. Van den Broeck, J.M.R. Parrondo, R. Toral, “Noise-induced Nonequilibrium Phase Transition”, Phys. Rev. Lett. 73 (1994) 3395.

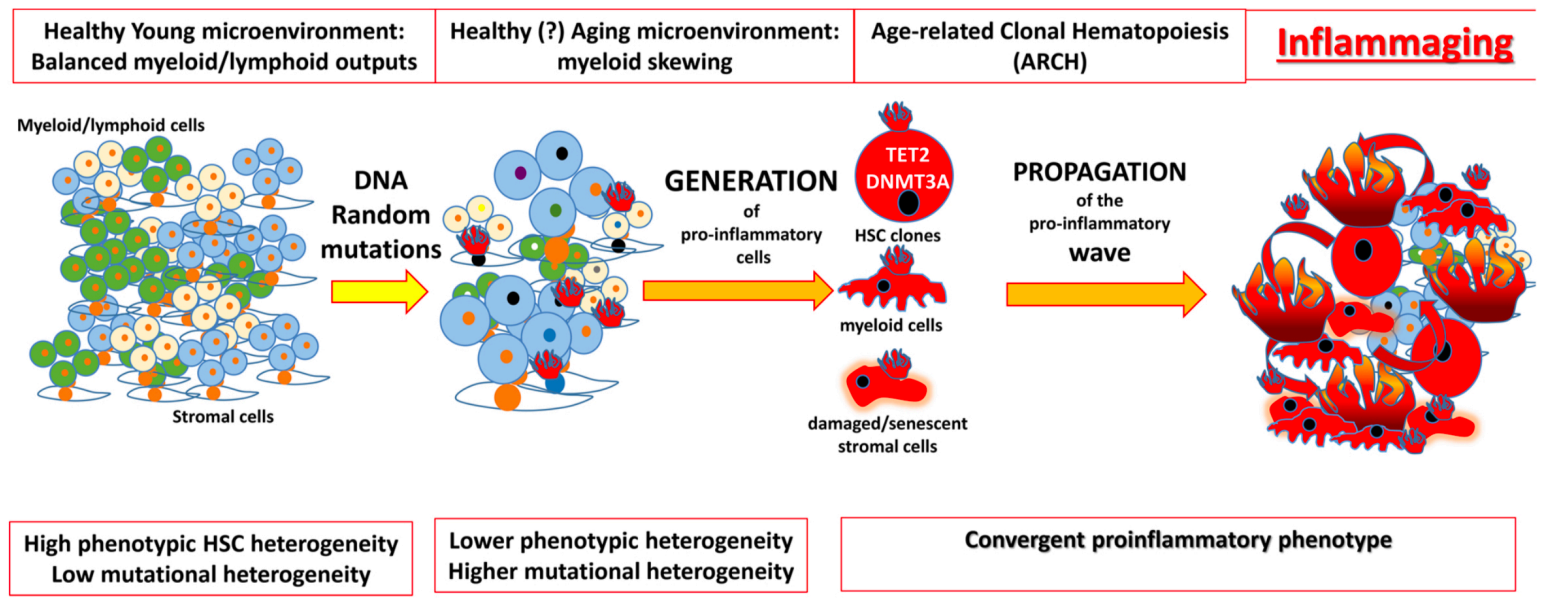

Fig. 1. Inflamm-aging of the bone marrow. Aging is characterized by the accumulation of random mutations in the DNA (increased mutation heterogeneity). Parallel to this phenomenon, the phenotypic diversity of HSC decreases owing to the progressive skewing towards the myeloid lineage (predominance of HSC myeloid skewed clones) and by the emergence of oligoclones that carry definite mutations (e.g. DNMT3A, TET2). As aging increases, expanded HSC clones promote the onset of a pro-inflammatory environment. At the same time, stromal cells activate the pro-inflammatory DNA damage response and ultimately undergo senescence. By interacting with myeloid cells, they propagate the pro-inflammatory signal along the tissue. In the final picture, the bone-marrow homeostatic regulation is taken over by pro-inflammatory HSC clones, stromal and myeloid cells. 


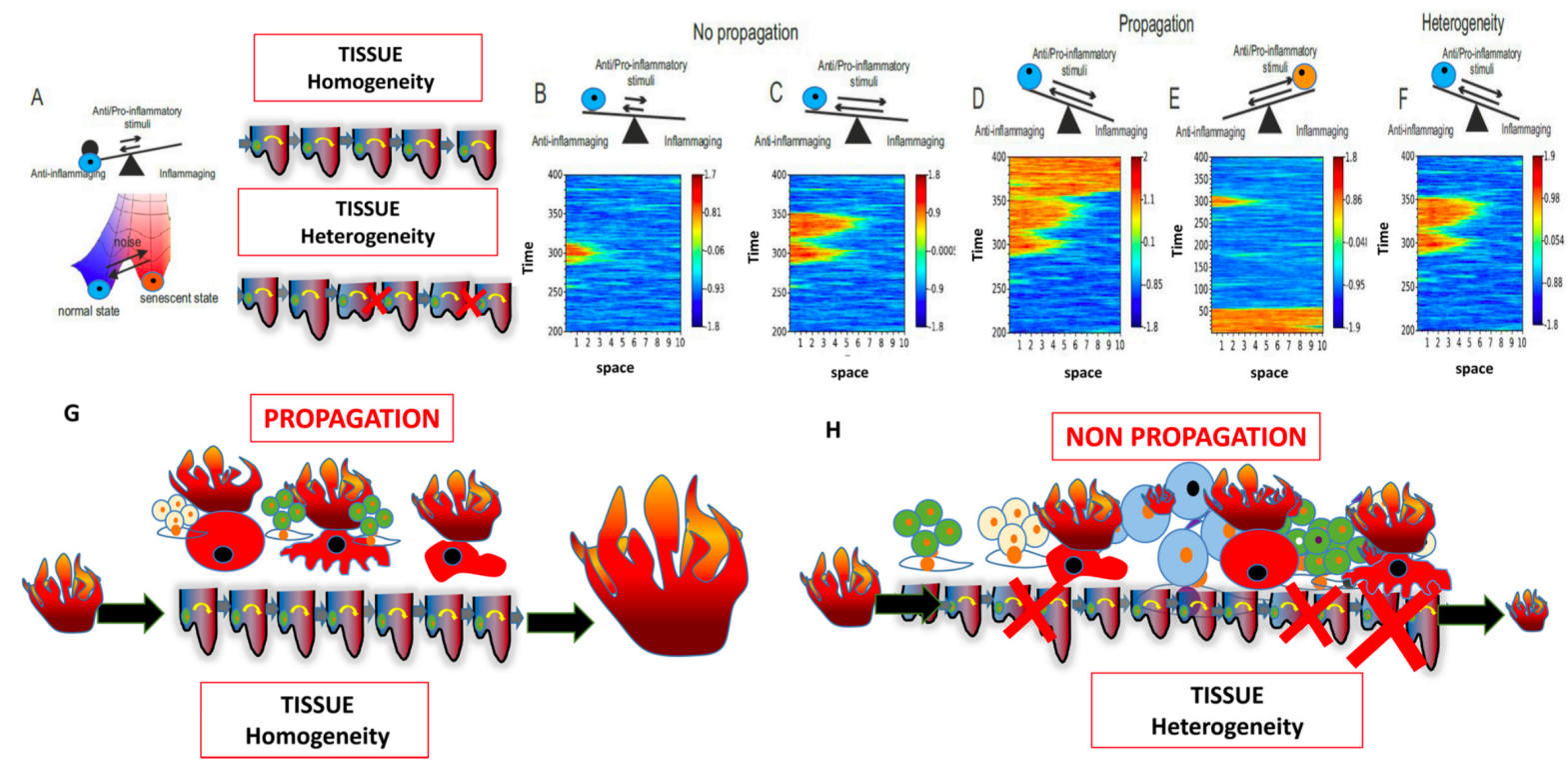

Fig. 2. Mathematical model of noise and tissue homogeneity/heterogeneity driven inflammaging. (Panel A) Chemical potential landscape of a cell with two metastable states, normal (left) and pro-inflammatory/senescent (right). The color code of the state of cells spans from blue (norm) to red (pro-inflammatory senescence status). Cells are aligned in one dimension and exchanging signals with neighbors. Cell interaction and noise from pro- and anti-inflammatory signals induce transitions between the states. Tissue homogeneity is represented by an array of cells that have the same bias of the potential landscape (Panels B-E). Tissue heterogeneity is represented by an array of cells with different bias of the potential landscape (Panel F). Assume that the noise is weak enough so that each cell remains in the "normal" meta-stable state for a long time. Eventually, fluctuations must switch one of the cells to the pro-inflammatory/senescent state. The response by the other cells to this transition depends on the parameters of the model. When the bias towards the pro-inflammatory/senescent state is only slight, the range of propagation of inflammation is sensitive to the amplitude of noise and can be substantially increased by it, although the system may finally resume the initial "normal" state, compare Panel B and Panel C. Expectantly, a greater bias towards the pro-inflammatory/senescent state favors the system-wide transition into it (Panel D), while a bias towards means that the inflamed area typically fades out, giving way to the "normal" (stronger) state (Panel E). In Panel F, we make use of the same noise and initial conditions as in Panel D, when local inflammatory process spreads over the whole system, but assign random values to the bias towards the pro-inflammatory/senescent state, keeping the same average (Tissue heterogeneity). We observe that the introduced heterogeneity detriments the propagation of the inflammatory front (Panel F). In this system of interacting bistable elements one can easily observe propagation of inflammation or anti-inflammation state. The resulting "winning" state will depend on the potential function, levels of stochasticity, interaction strength and heterogeneity. Nevertheless, it is not necessary that this phenomenological system is intrinsically bistable. Indeed, bistability can be induced by stochasticity as a manifestation of collective dynamics and result from the interplay between stochasticity and coupling in an ensemble of interacting elements in "nonequibrium" systems [28]. 


\section{Inflammaging}

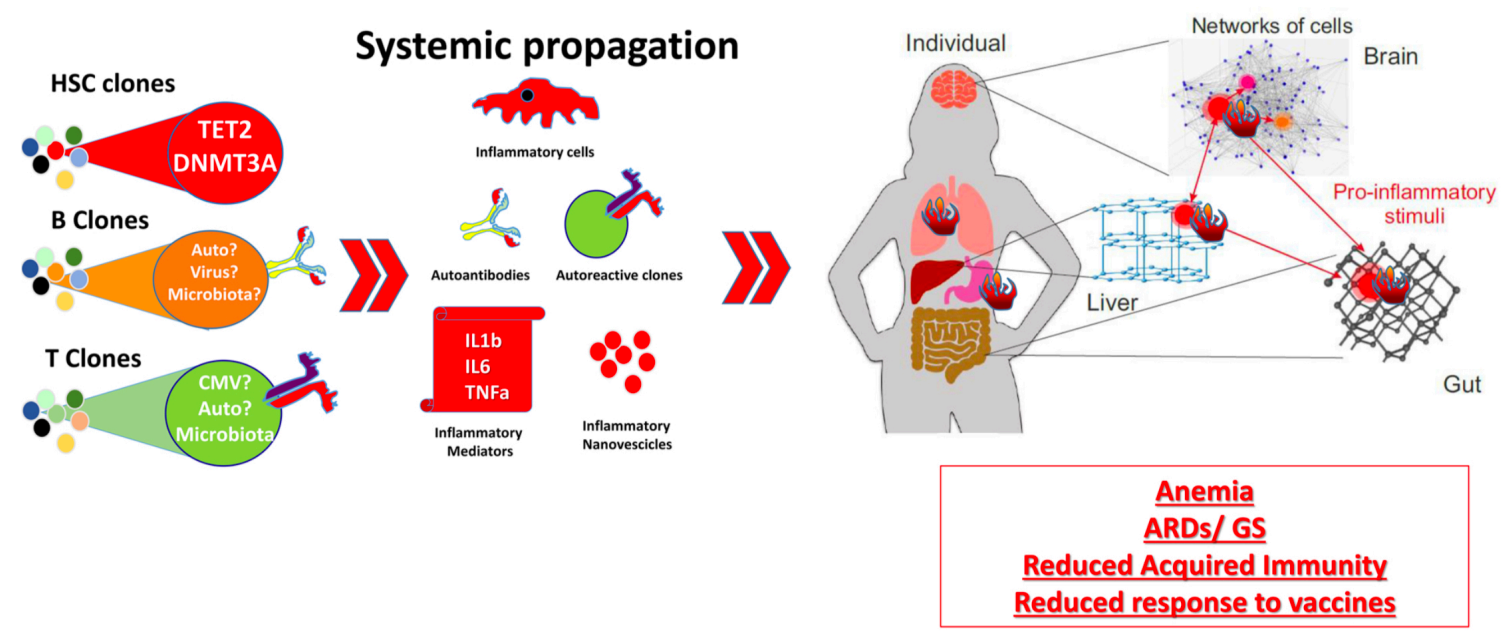

Fig. 3. Expansion of hematopoietic/immune system clones depending spreading of inflammatory signals. Similarly, to the bone marrow the immune system of aged people is progressively fulfilled and dominated by $\mathrm{T}$ and $\mathrm{B}$ cell clones, which are likely to represent an adaptation (or remodeling) rather than a simple derangement of the senescent immune system. Indeed, these T/B clones represent the result of the chronic attrition of antigens that can span from chronic/latent infections, microbiota and autoantigens. These latter may arise de novo during aging as a derangement of self recognition. Expanded Hematopoietic/immune system clones are likely to spread inflammatory signal (cell, nanovesicles and soluble mediators) to the systemic level. 


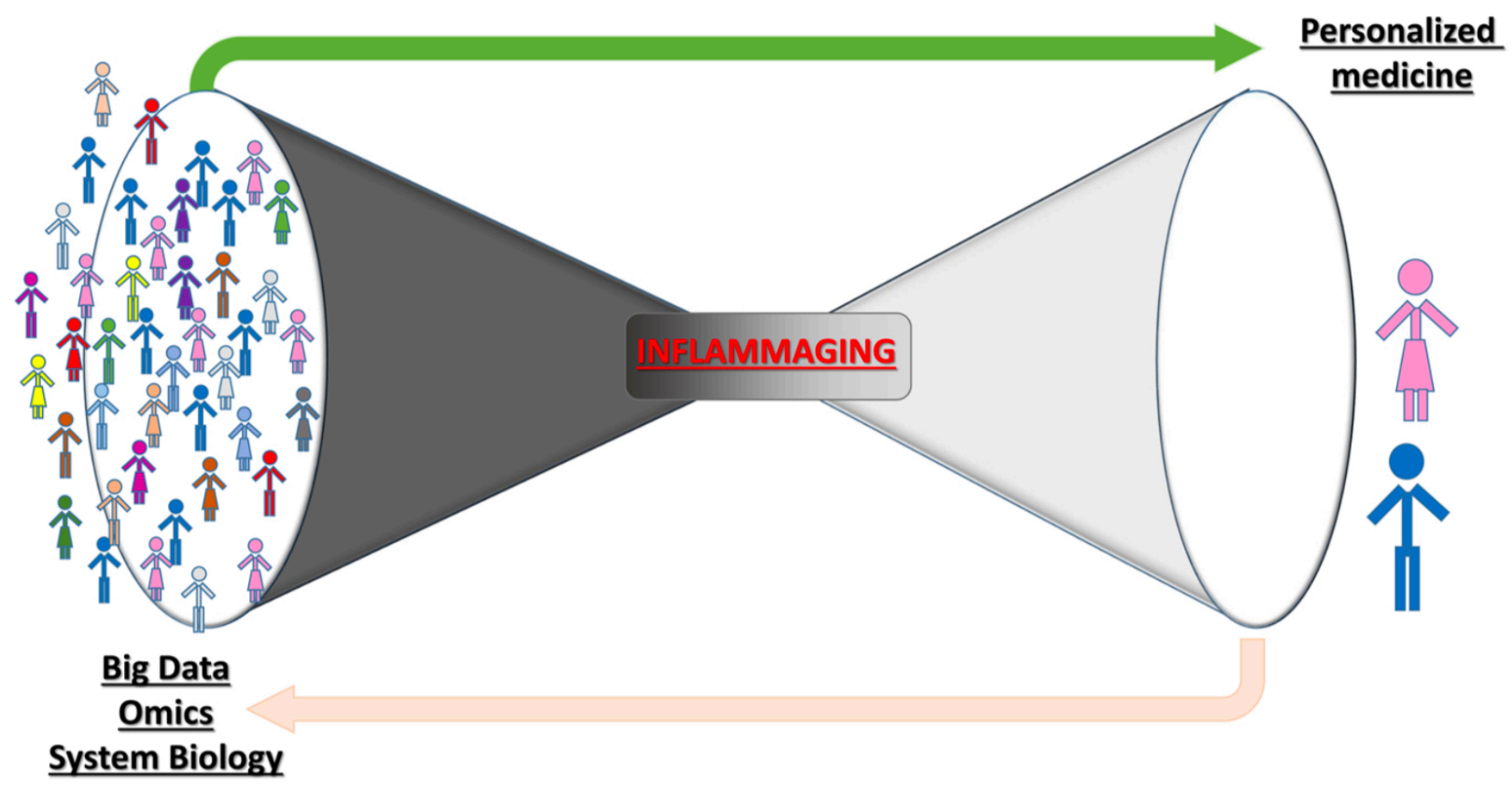

Fig. 4. Inflammaging: from BIG DATA to tailored therapeutic strategies. Representative picture describing the importance of BIG DATA/OMICs multidisciplinary approach analysis in population studies aimed at identify Biomarkers of Inflammaging. The final goal is to define diagnostic tools able to setting tailored therapeutic strategies for age related diseases burdens. 